\title{
Acute encephalopathy due to occupational exposure to arsenic
}

\author{
W S BECKETT, J L MOORE, J P KEOGH, AND MARGIT L BLEECKER \\ From the Divisions of Occupational Medicine, Neurology, and Psychiatry, Johns Hopkins Medical Institutions, \\ Baltimore, MD, USA
}

Before the advent of penicillin, acute haemorrhagic encephalitis was described as a complication of parenteral arsphenamine treatment for syphillis. ${ }^{12}$ Occupational exposure to toxic amounts of arsenic in the workplace has most commonly resulted in the appearance of peripheral neuropathy, rash, or gastrointestinal symptoms. The following case describes acute encephalopathy after occupational exposure to arsenic.

A 50 year old chemical plant engineer presented with delirium. At the age of 22 while in the armed forces he had been briefly admitted to hospital with a psychiatric illness that he described as being related to the stresses of his job. For over 20 years he had worked intermittently with a smelting process in which antimony is purified from antimony ore; industrial hygiene monitoring of the process had detected sulphur, arsenic, copper, iron, and lead in the ore and contaminating the finished product. Five years before admission, with complaints of diarrhoea, cough, rash, and feelings of anger and suspicion, he had consulted a physician who measured urine arsenic at $21 \mu \mathrm{g} / 1$ (normal 0-20); the symptoms resolved without treatment over several days, and he returned to work. He had no history of alcoholism or recent drug use. His wife of 17 years had noted a gradual change in his personality over several years accompanied by difficulties with memory and marked by episodes of suspiciousness and marital conflict.

During the week before presentation he had spent several days working within the smelting furnace, failing to use the required respiratory protection. On the day before presentation, he experienced severe nausea, vomiting, and diarrhoea, and awoke from sleep with hallucinations, disorientation, and agitation. He sought medical attention and did not return to work; his mental status gradually improved. One month after the end of his exposure, he again became agitated and was admitted to the hospital where examination was remarkable only for delirium, which rap-

Accepted 1 July 1985 idly resolved. He continued to exhibit a rapidly fluctuating mental state with prominent agitation and emotional lability. Blood counts, serum electrolytes, 8 calcium, phosphorus, glucose, BUN, T-4, and electro- 0 cardiogram results were normal; a CT scan of the head showed only a slightly asymmetric right lateral $c$ ventricle. An EEG two days after admission showed only mild slowing of the alpha rate, considered to be within normal limits. Urine arsenic was $41 \mu \mathrm{g} / 1$ (normal occupational exposure $<30 \mu \mathrm{g} / 1)$. Urinary anti- $\vec{\emptyset}$ mony, lead, and selenium were within expected limits for those occupationally exposed. No urine mercury 0 could be detected, and urine manganese was within normal limits. Arsenic in the nuchal hair was $4.0 \mu \mathrm{g} / \mathrm{g}$ in the distal shafts (normal 0.03 to $0.32 \mu \mathrm{g} / \mathrm{g}$ ) and $5 \cdot 1 \mu \mathrm{g} / \mathrm{g}$ in the proximal shafts. Nerve conduction $\stackrel{\circ}{\varnothing}$ studies of the feet detected no sensory deficit. His $\stackrel{\varrho}{\Rightarrow}$ mental state improved steadily over several days $\overrightarrow{\overrightarrow{0}}$ before the initiation of chelation treatment

During a nine day course of chelation with BAL, urine arsenic excretion fell to undetectable levels for several days, then returned to levels seen before the $\stackrel{\odot}{\Phi}$ start of chelation. His mental state continued to $?$ improve during treatment. After his discharge from $\frac{5}{3}$ the hospital, his wife noted persistent memory loss and more pronounced changes in personality and $\delta$ behaviour since the episode of delirium. Three weeks after the end of BAL treatment he became severely 옥 agitated without delirium. The agitation resolved rap- $\rightarrow$ idly within 24 hours, before the initiation of a second course of BAL chelation. Psychological testing detected deficits in his ability to learn new information, by contrast with a good performance when pre- $\tilde{N}$ viously learned information was required for the task.

Chronic occupational arsenic exposure can occur $\bar{\sigma}$ through inhalation, ingestion, or absorption through 0 the skin, and has most frequently been associated $\frac{\Phi}{\Phi}$ with chronic mixed motor and sensory poly- $\stackrel{?}{?}$ neuropathy after prolonged low level exposure. Epi- 7 demiological studies have shown an increased inci- $\stackrel{\circ}{\Phi}$ dence of skin and lung cancer in those exposed.

Clinicopathological correlation of arsenic intoxi- $\stackrel{\mathbb{Q}}{\mathscr{Q}}$ 
cations with encephalopathy have established haemorrhagic cerebral infarction as part of the course in fatal poisoning. These necropsy studies arose from cases of accidental overdose with parenteral or oral arsenicals used for their antimicrobial effects. Study of the brain from a fatal case in a patient treated with an oral arsenical showed multiple areas of microcavitary necrosis with areas of microglial proliferation, foci of perivascular red cells and histiocytes with extravasation of blood, and large ring haemorrhages in the white matter. ${ }^{2}$ Other series of fatal acute arsenic encephalitis have described similar areas of necrosis and haemorrhage, most frequently in the white matter, but in some cases affecting almost all of the brain. ${ }^{3}$ Non-haemorrhagic cases may show widespread cortical areas of destruction or myelin breakdown. ${ }^{4}$

This patient's early gastrointestinal symptoms were typical of acute arsenic intoxication, but his illness was unusual in the duration and the recurrence of encephalopathy ${ }^{5}$ and in the absence of peripheral neuropathy as a sequel of chronic low level exposure. His first urinary arsenic concentrations, taken more than a week after the end of exposure, were lower than those usually described in acute intoxications. Acute exposure to arsenic commonly results in a rapid increase in urinary excretion which then rapidly returns to normal. ${ }^{6}$

The two episodes of delirium resolved rapidly before institution of BAL therapy, and although further improvement occurred simultaneously with chelation, there were no unequivocal indicators of a beneficial effect of this treatment. Review of previous reports of chelation therapy for arsenical encepha- lopathy also provide inadequate data to determine clinical benefit. His course confirms a previous observation concerning arsenic poisoning: the history of exposure to arsenic may be more important for diagnosis than blood or tissue levels. ${ }^{2}$ This patient's history of a remote psychiatric illness may indicate an abnormal susceptibility to the central nervous system effects of arsenic. His longstanding behavioural changes in association with chronic recurrent exposures may also indicate an increased susceptibiliy to the acute insult of an unusually heavy exposure.

Requests for reprints to: $\mathrm{Dr} W \mathrm{~W}$ Beckett, Occupational Medicine Program, Yale University School of Medicine, 333 Cedar St, New Haven, CT 06510.

\section{References}

${ }^{1}$ Glaser MA, Imerman CP, Imerman SW. So-called hemorrhagic encephalitis and myelitis secondary to intravenous arsphenamins. Am J Med Sci 1935;189:64-79.

${ }^{2}$ Cole M, Scheulein M, Kerwin D. Arsenical encephalopathy due to use of Milibis. Arch Int Med 1966;117:706-11.

${ }^{3}$ Call RD, Gunn FD. Arsenical encephalopathy. Arch Pathol 1949;48:119-48.

${ }^{4}$ Goetz C, Klawans H, Cohen M. Neurotoxic agents. In: Baker B, Baker LM, eds. Clinical neurology. Philadelphia: Harper and Rowe, 1982.

${ }^{5}$ Greenhouse A. Heavy metals and the nervous system. Clinical Neuropharmacology 1982;5:45-92.

${ }^{6}$ Goldstein NP, McCall JT, Dyck PJ. Metal neuropathy. In: Dyck PJ, Thomas PK, Lambert EH, eds. Peripheral neuropathy. Philadelphia: WB Saunders Co, 1977:1227-62. 